\title{
Higgs decay into two leptons and a photon revisited
}

\author{
Aliaksei Kachanovich ${ }^{a, *}$ \\ ${ }^{a} T T P, K I T$, \\ Wolfgang-Gaede-Straße 1, Karlsruhe, Germany \\ E-mail: aliaksei.kachanovich@kit.edu
}

I present new results for the Standard-Model predictions of the differential decay rates for $H \rightarrow$ $\ell^{+} \ell^{-} \gamma$, where $\ell=e, \mu$, and the forward-backward asymmetries defined in terms of the flight direction of the photon corresponding to the lepton momenta. The results depend on the selection criteria for the energies and invariant masses (i.e. the cuts) of the final state particles. For standard choices of these cuts the branching ratios $B(H \rightarrow e \bar{e} \gamma)=5.8 \cdot 10^{-5}$ and $B(H \rightarrow \mu \bar{\mu} \gamma)=6.4 \cdot 10^{-5}$ as well as the forward-backward asymmetries $\mathcal{A}_{\mathrm{FB}}^{(e)}=0.343$ and $\mathcal{A}_{\mathrm{FB}}^{(\mu)}=0.255$ have been found.

40th International Conference on High Energy physics - ICHEP2020

July 28 - August 6, 2020

Prague, Czech Republic (virtual meeting)

${ }^{*}$ Speaker 


\section{Motivation}

The Standard Model (SM) is a very successful but incomplete theory. Theories of new physics typically involve extensions of the minimal Higgs sector of the SM and precision measurements of production and decay of the $125-\mathrm{GeV}$ Higgs particle $h$ can eventually reveal virtual effects of such an extended Higgs sector. Couplings of $h$ can be modified by the mixing of the SM Higgs field with another scalar field, as in e.g. Higgs portal models of Dark Matter (see [1-3] for recent studies). Another possible impact of new physics — not necessarily related to extended Higgs sectors - is the modification of $h$-couplings through loop effects involving new particles. Such effects are best detectable in processes which are suppressed in the SM. This situation occurs if the SM process itself is loop-dominated or involves a tiny tree-level coupling. The process $h \rightarrow \ell \bar{\ell} \gamma$, with $\ell$ representing an electron $e$ or muon $\mu$, are in this category. Discrepancies between different results in the literature [4-8] have triggered the new study in Ref. [9] which I present here.

All contributions to the process $h \rightarrow \ell \bar{\ell}$ are proportional to the Yukawa coupling, so that the leptons in the final state always have opposite chirality, i.e. if one lepton is right-handed the second one is always left-handed. By contrast, in the process $h \rightarrow \ell \bar{\ell} \gamma$ both leptons can be in the same helicity state. The dominant contribution in the case of the final state $\ell_{L(R)} \bar{\ell}_{L(R)} \gamma$ comes from the one-loop level and is not vanishing even for $m_{\ell}=0$. As a result, the rate for the process $h \rightarrow e \bar{e} \gamma$ is much larger than the rate of $h \rightarrow e \bar{e}$, while the analogous rates for muons are of the same order of magnitude $\left(\mathcal{B}(h \rightarrow \mu \bar{\mu} \gamma)=6.7 \times 10^{-5}\right.$ vs. $\left.\mathcal{B}(h \rightarrow \mu \bar{\mu})=2 \times 10^{-4}\right)$.

\section{Calculations}

The Mathematica package FeynArts [10, 11] finds 436 one-loop diagrams contributing to $h \rightarrow \ell \bar{\ell} \gamma$. Ignoring those involving the small lepton Yukawa coupling reduces the number of diagrams to 119 , which can be categorised by their topologies and the type of propagator to which the lepton pair are coupled into 13 classes shown in Fig. 1. We perform the evaluation of these diagrams with the help of the FeynCalc package [12-14] in a general $R_{\xi}$ gauge. For some analytical checks we used Package- $X$ [15] which can be linked to FeynCalc via the FeynHelpers add-on [16]. One of the goals of this calculation was to find gauge-independent classes of diagrams. Only Classes 10 and 11 (see Fig. 1) are gauge independent, while all other diagrams must be added for another gauge independent subset. For photon, Z-, and W-boson propagators three different gauge parameters $\xi_{\gamma}, \xi_{Z}$, and $\xi_{W}$ are used. The cancellation of the parameters $\xi_{\gamma}$ and $\xi_{Z}$ happens after the summation of all diagrams. For the cancellation of the parameter $\xi_{W}$, it is crucial that the W-boson mass $m_{W}$ and the Z-boson mass $m_{Z}$ fulfill the Weinberg relation

$$
e^{2} / g_{2}^{2}=\sin ^{2} \theta_{W}=1-m_{W}^{2} / m_{Z}^{2},
$$

where $e, g_{2}$ and $\theta_{W}$ are the electromagnetic and weak coupling constants and the weak mixing angle, respectively. This condition restricts the choice of the value of the fine structure constant $\alpha=e^{2} /(4 \pi)$ after $g_{2}$ is fixed in terms of $M_{W}$ by the experimental value of the Fermi constant $G_{F}$.

The amplitude satisfies the QED Ward identity and can be written as:

$$
\begin{aligned}
\mathcal{A}_{\text {loop }} & =\left[\left(k_{\mu} p_{1 v}-g_{\mu \nu} k \cdot p_{1}\right) \bar{u}\left(p_{2}\right)\left(a_{1} \gamma^{\mu} P_{R}+b_{1} \gamma^{\mu} P_{L}\right) v\left(p_{1}\right)\right. \\
& \left.+\left(k_{\mu} p_{2 v}-g_{\mu \nu} k \cdot p_{2}\right) \bar{u}\left(p_{2}\right)\left(a_{2} \gamma^{\mu} P_{R}+b_{2} \gamma^{\mu} P_{L}\right) v\left(p_{1}\right)\right] \varepsilon^{v^{*}}(k),
\end{aligned}
$$




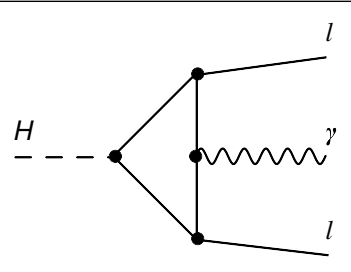

(a)

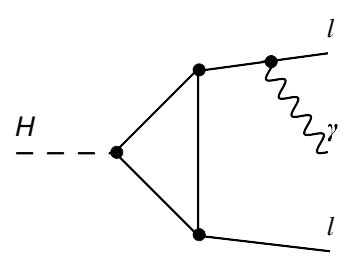

(b)

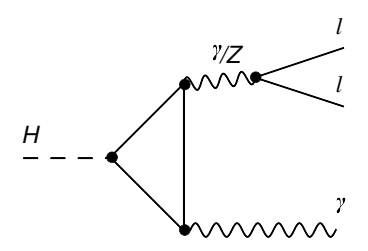

(c)

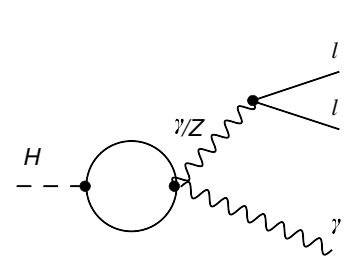

(d)

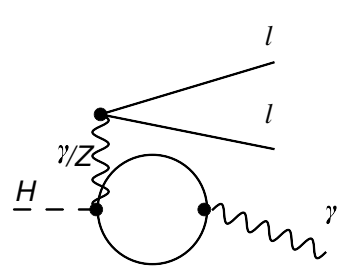

(e)

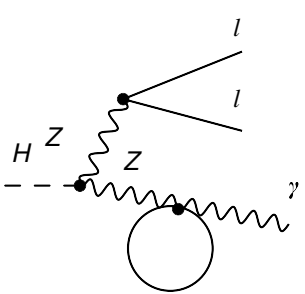

(f)

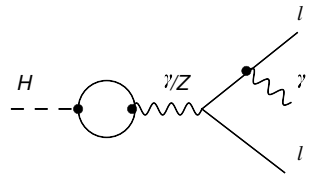

(g)

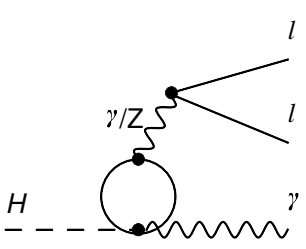

(h)

Figure 1: These Feynman diagrams represent different classes of diagrams which contributes to the process $H \rightarrow \ell \bar{\ell} \gamma$ at one-loop level. The solid lines without label denote generic SM propagators to be replaced by all possibilities permitted by the SM Feynman rules (any valid SM propagators with appropriate vertices). Diagrams a) represent Class 1, b) Class 2, c) Classes 3 and 4, d) Classes 5 and 6, e) Classes 7 and 8, f) Class 9, g) Classes 10 and 11, h) Classes 12 and 13. If two classes are mentioned, they refer to $\gamma$ and $Z$.

where $k_{\mu}, p_{1 \mu}$, and $p_{2 \mu}$ are photon, lepton, and anti-lepton momenta, respectively. The coefficients $a_{1}, a_{2}, b_{1}$, and $b_{2}$ can be found in Ref. [9], where they are presented in a way suited for numerical implementation.

In the case of the electron, the tree-level contribution can be completely neglected. For the muon, the tree-level contribution is large enough to be relevant, with the amplitude reading

$$
\mathcal{A}_{\text {tree }}=-\frac{e^{2} m_{\ell} \varepsilon_{v}^{*}(k)}{2 m_{W} \sin \theta_{W}}\left[\frac{\bar{u}\left(p_{1}\right)\left(\gamma^{v} \not k+2 p_{1}^{v}\right) v\left(p_{2}\right)}{t-m_{\ell}^{2}}-\frac{\bar{u}\left(p_{1}\right)\left(k \gamma^{v}+2 p_{2}^{v}\right) v\left(p_{2}\right)}{u-m_{\ell}^{2}}\right] \text {, }
$$

where squared invariant masses are denoted by the Mandelstam variables $s=\left(p_{1}+p_{2}\right)^{2}, t=$ $\left(p_{1}+k\right)^{2}$, and $u=\left(p_{2}+k\right)^{2}$. To regulate the Z-boson pole, we used a Breit-Wigner propagator in the diagram classes (c-f) and (h) shown in Fig. 1. To this end our result with full dependence on the gauge parameters helped us to avoid spurious gauge dependences related to the fact that the $h \rightarrow Z \gamma$ vertex is gauge-dependent once the $Z$ is taken off-shell. 


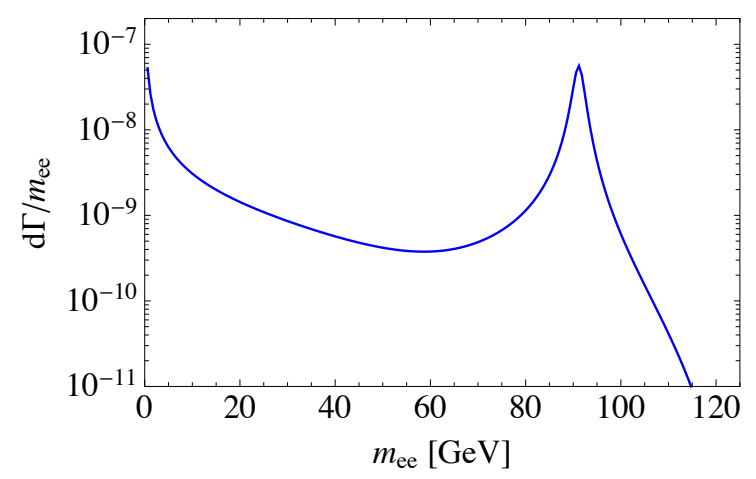

(a)

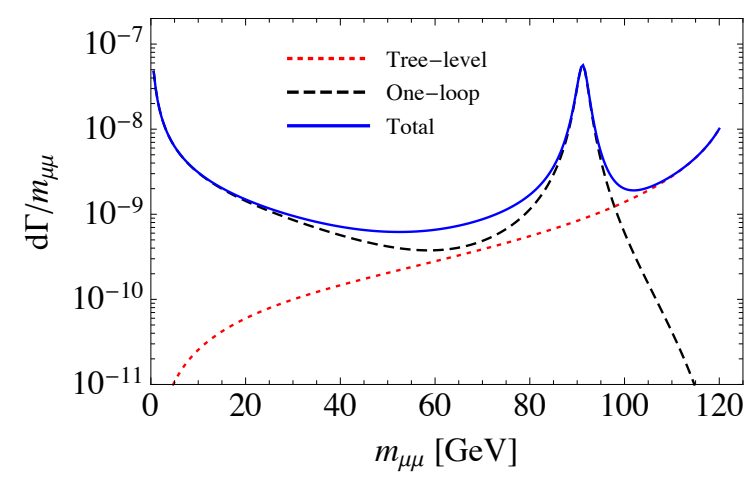

(b)

Figure 2: Differential decay rate for (a) electrons and (b) muons with respect to the invariant dilepton mass. The total, one-loop, and tree-level contributions are denoted by solid blue, black dashed, and red dotted lines, respectively. For the case of electrons, the tree-level contribution is negligible. The cut $E_{\gamma, \min }=5 \mathrm{GeV}$ has been implemented.

\section{Results}

Our results for the differential decay rates for electrons and muons, shown in Fig. 2, have been obtained using the following values of input parameters:

$$
\begin{gathered}
m_{W}=80.379 \mathrm{GeV}, \quad m_{Z}=91.1876 \mathrm{GeV}, \quad \sin ^{2} \theta_{W}=1-\frac{m_{W}^{2}}{m_{Z}^{2}}=0.223013, \\
m_{t}=173.1 \mathrm{GeV}, \quad m_{H}=125.1 \mathrm{GeV}, \quad m_{e}=5.110 \times 10^{-4} \mathrm{GeV}, \quad m_{\mu}=0.106 \mathrm{GeV}, \\
G_{F}=1.1663787 \times 10^{-5} \mathrm{GeV}^{-2}, \quad \alpha^{-1}=\frac{\pi}{\sqrt{2} G_{F} m_{W}^{2} \sin ^{2} \theta_{W}}=132.184,
\end{gathered}
$$

The choice of the value for the inverse fine structure constant is dictated by Eq. 1 and crucial for the vanishing of the gauge dependence. Radiative corrections lead to the more familiar value $\alpha^{-1} \simeq 128$, but the proper use of this value would require the inclusion of higher-order corrections to the decay amplitude as well.

The full decay rate has been evaluated after employing the kinematics cuts of Ref. [5, 6], namely:

$$
\begin{aligned}
& s, t, u>\left(0.1 m_{H}\right)^{2}, \quad E_{\gamma}>5 \mathrm{GeV}, \\
& \left(E_{1}>7 \mathrm{GeV}, \quad E_{2}>25 \mathrm{GeV}\right) \quad \text { or } \quad\left(E_{1}>25 \mathrm{GeV}, \quad E_{2}>7 \mathrm{GeV}\right) .
\end{aligned}
$$

We also predict the differential decay distribution with respect to the angle between lepton and photon, $\theta^{(\ell)}$. The dependence of the decay rate on $\cos \theta^{(\ell)}$ is shown in Fig. 4. The forward-backward asymmetry is defined as

$$
\mathcal{A}_{l, \mathrm{FB}}=\frac{\int_{-1}^{0} \frac{d \Gamma}{d \cos \theta_{l}}-\int_{0}^{1} \frac{d \Gamma}{d \cos \theta_{l}}}{\int_{-1}^{0} \frac{d \Gamma}{d \cos \theta_{l}}+\int_{0}^{1} \frac{d \Gamma}{d \cos \theta_{l}}},
$$




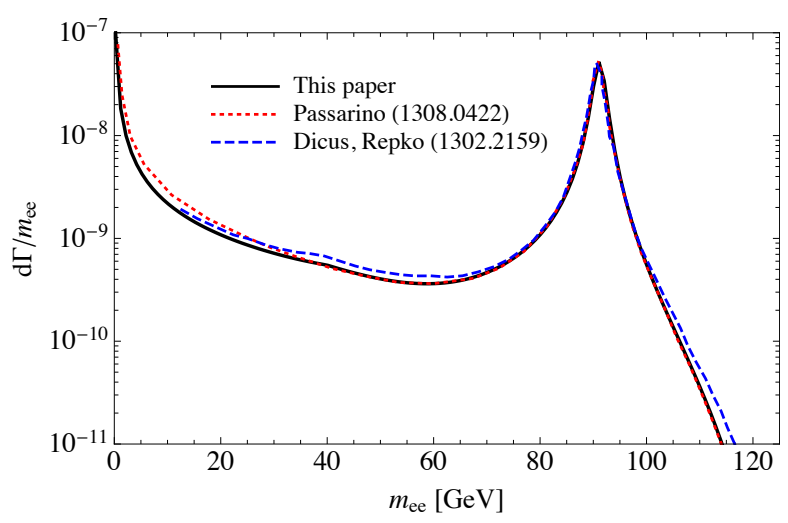

Figure 3: Differential decay rate with respect to the invariant dielectron mass. Our new result is denoted by a black solid line, while the results of Refs. [6] and [5] are denoted by blue long-dashed and red short-dashed lines, respectively.

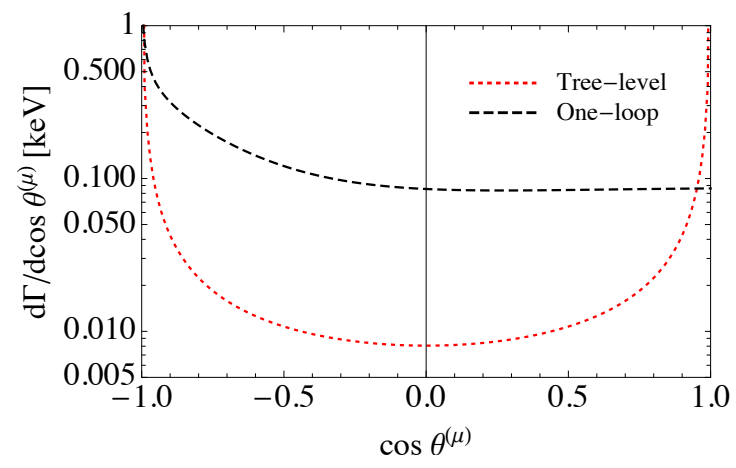

Figure 4: Differential decay rate with respect to $\cos \theta^{(\mu)}$, where $\theta^{(\mu)}$ is the angle between the photon and one of the leptons in the Higgs boson rest frame. The cuts $m_{\mu \mu}>0.1 m_{H}$ and $E_{\gamma, \min }=5 \mathrm{GeV}$ have been implemented.

where cuts $m_{\ell \ell}>0.1 m_{H}$ and $E_{\gamma, \min }=5 \mathrm{GeV}$ have been used. We find:

$$
\mathcal{A}_{e, \mathrm{FB}}=0.343, \quad \mathcal{A}_{\mu, \mathrm{FB}}=0.255 .
$$

\section{Conclusions}

A novel analysis of the SM predictions for various observables in the decays $h \rightarrow \ell \bar{\ell} \gamma$ has been presented. We have clarified discrepancies in the literature (see Fig. 3) and the issue of a gaugeindependent implementation of a finite $Z$ width. Furthermore, a gauge-independent result requires that the weak mixing angle defined in terms of the gauge couplings fulfills the on-shell-scheme definition $m_{W}=\cos \theta_{W} m_{Z}$.

For typical choices of cuts we find the branching ratios $B(h \rightarrow e \bar{e} \gamma)=5.8 \cdot 10^{-5}$ and $B(h \rightarrow \mu \bar{\mu} \gamma)=6.4 \cdot 10^{-5}$ as well as the forward-backward asymmetries $\mathcal{A}_{\mathrm{FB}}^{(e)}=0.343$ and 
$\mathcal{A}_{\mathrm{FB}}^{(\mu)}=0.255$. We provide analytic results in Appendix A of Ref. [9] and in the corresponding ancillary files.

\section{Acknowledgements}

I thank Ulrich Nierste and Ivan Nišandžić for the enjoyable collaboration on the presented work and acknowledge the support from the doctoral school KSETA and the Graduate School Scholarship Programme of the German Academic Exchange Service (DAAD).

\section{References}

[1] G. Krnjaic, Phys. Rev. D 94 (2016) no.7, 073009 [arXiv:1512.04119 [hep-ph]].

[2] A. Kachanovich, U. Nierste and I. Nišandžić, Eur. Phys. J. C 80 (2020) no.7, 669 doi:10.1140/epjc/s10052-020-8240-z [arXiv:2003.01788 [hep-ph]].

[3] A. Filimonova, R. Schäfer and S. Westhoff, Phys. Rev. D 101 (2020) no.9, 095006 [arXiv: 1911.03490 [hep-ph]].

[4] L. B. Chen, C. F. Qiao and R. L. Zhu, Phys. Lett. B 726 (2013), 306-311 [erratum: Phys. Lett. B 808 (2020), 135629] [arXiv:1211.6058 [hep-ph]].

[5] G. Passarino, Phys. Lett. B 727 (2013), 424-431 [arXiv:1308.0422 [hep-ph]].

[6] D. A. Dicus and W. W. Repko, Phys. Rev. D 87 (2013) no.7, 077301 [arXiv:1302.2159 [hep-ph]].

[7] Y. Sun, H. R. Chang and D. N. Gao, JHEP 05 (2013), 061 [arXiv:1303.2230 [hep-ph]].

[8] T. Han and X. Wang, JHEP 10 (2017), 036 [arXiv:1704.00790 [hep-ph]].

[9] A. Kachanovich, U. Nierste and I. Nišandžić, Phys. Rev. D 101 (2020) no.7, 073003 [arXiv:2001.06516 [hep-ph]].

[10] J. Kublbeck, M. Bohm and A. Denner, Comput. Phys. Commun. 60 (1990), 165-180

[11] T. Hahn, Comput. Phys. Commun. 140 (2001), 418-431 [arXiv:hep-ph/0012260 [hep-ph]].

[12] V. Shtabovenko, R. Mertig and F. Orellana, Comput. Phys. Commun. 256 (2020), 107478 [arXiv:2001.04407 [hep-ph]].

[13] V. Shtabovenko, R. Mertig and F. Orellana, Comput. Phys. Commun. 207 (2016), $432-444$ [arXiv:1601.01167 [hep-ph]].

[14] R. Mertig, M. Bohm and A. Denner, Comput. Phys. Commun. 64 (1991), 345-359

[15] H. H. Patel, Comput. Phys. Commun. 197 (2015), 276-290 [arXiv:1503.01469 [hep-ph]].

[16] V. Shtabovenko, "FeynHelpers: Connecting FeynCalc to FIRE and Package-X," Comput. Phys. Commun. 218 (2017), 48-65 [arXiv:1611.06793 [physics.comp-ph]]. 